\title{
Post-intensive care syndrome
}

\author{
A crash course for general practice
}

\section{Pierre Berger, David Braude}

This article is the first in a two-part series, outlining the key facets of post-intensive care syndrome to facilitate early diagnosis and appropriate treatment.

\section{Background}

Post-intensive care syndrome (PICS) refers to a constellation of cognitive, psychiatric and physical symptoms experienced by patients during and following a period of critical illness. As many as $50 \%$ of intensive care unit (ICU) survivors are affected, and symptoms can persist for months to years. When psychological symptoms are experienced by patients' loved ones, this is termed PICS-family (PICS-F).

\section{Objective}

The aim of this article is to summarise the key facets of PICS and PICS-F with a focus on incidence and pathophysiology.

\section{Discussion}

The amalgam of symptoms in PICS has a profound impact on the quality of life of affected ICU survivors. The number of patients with PICS is expected to rise considerably as a result of the COVID-19 pandemic. Primary care practitioners are ideally situated to assist in early diagnosis and treatment.
POST-INTENSIVE CARE SYNDROME (PICS) refers to a constellation of cognitive, psychiatric and physical symptoms experienced by patients during and following a period of critical illness. As many as $50 \%$ of intensive care unit (ICU) survivors are affected, and symptoms can persist for months to years. ${ }^{1}$ When psychological symptoms are experienced by patients' loved ones, this is termed PICS-family (PICS-F).

The COVID-19 pandemic is expected to increase the number of patients with these syndromes.

\section{Cognitive features}

Impaired cognition after critical illness can be significant, with patients' memory, attention, higher executive functions and visuospatial abilities diminished. ${ }^{1}$

In the 2013 BRAIN-ICU (Bringing to light the Risk factors And Incidence of Neuropsychological dysfunction in ICU survivors) study, $34 \%$ of ICU survivors displayed global cognition scores similar to those seen in patients with moderate traumatic brain injury at 12-month follow-up. ${ }^{2}$ This long-term impairment was independent of age and co-existing medical conditions, with duration of delirium in ICU identified as the key risk factor. The inflammation, cerebral atrophy and reduced white matter integrity associated with delirium are hypothesised to underpin this process. ${ }^{3}$

\section{Physical features}

ICU-acquired weakness is a serious complication affecting up to $50 \%$ of patients admitted to the ICU for at least one week. ${ }^{4}$ It is characterised by diffuse symmetrical limb and/or diaphragmatic weakness and is associated with increased mortality and long-term functional impairment. ${ }^{5}$ Major risk factors are prolonged mechanical ventilation, sepsis, organ failure and immobility. ${ }^{5}$

Undernutrition is another contributing factor, with ICU patients typically receiving only $60-80 \%$ of their enteral energy requirements. ${ }^{6}$ Rapid loss of weight and muscle bulk is associated with decreased mobility, patient fatigue and increased hospital length of stay. ${ }^{7}$ Malnourishment also impairs immune function, increasing risk of nosocomial infection and mortality. ${ }^{7}$ Furthermore, critical illness can lead to vitamin D deficiency and bone hyperresorption, with ICU survivors exhibiting decreased bone mineral density and significantly elevated Fracture Risk Assessment Tool (FRAX) scores at one-year follow-up. ${ }^{8}$

Long-term pulmonary dysfunction is another key issue. Protracted mechanical ventilation increases the risk of ventilatorinduced lung injury, pneumonia and diaphragmatic myotrauma. Lung function, measured with spirometry, typically improves by six months but can remain compromised for as long as five years. ${ }^{1}$ 
The trauma of critical illness can result in debilitating chronic pain, with one-year prevalence estimated at up to $77 \% .{ }^{9}$ Studies examining health-related quality of life have consistently shown pain-related functional interference with activities of daily living at three and 12 months. ${ }^{9}$

Patients with physical sequelae of critical illness experience long-term impairment, and the consequent loss of agency can exacerbate psychological distress.

\section{Psychiatric features}

The psychological conditions affecting patients with PICS are anxiety, depression and post-traumatic stress disorder (PTSD), with a prevalence of $46 \%, 40 \%$ and $22 \%$, respectively. ${ }^{10}$ Critical illness is frequently distressing. Patients grapple with feelings of helplessness and their own mortality. Treatments are invasive, and commonly used medications can have significant neuropsychiatric side effects. Many patients experience sleep deprivation, thirst and pain. ${ }^{11}$ Patients with delirium experience frightening hallucinations and delusions, and these often persist as traumatic memories, which can impair emotional brain circuitry. The result is abnormal cognitive processing and a feeling of ongoing threat and vulnerability. ${ }^{12}$ Predisposing factors for psychological sequelae are younger age, female gender, pre-existing psychiatric conditions and socioeconomic variables such as unemployment and lower education level. ${ }^{1}$

\section{Quality of life}

The amalgam of physical, cognitive and psychological symptoms in PICS can profoundly limit ICU survivors' functional independence and societal integration. At one year, approximately $50 \%$ require daily caregiver assistance, and many are unable to drive, limiting community engagement. ${ }^{13}$ One-third never return to paid employment, and those who do frequently report a reduction in income. ${ }^{14}$ The associated financial burden is both a significant stressor and barrier to accessing rehabilitation services, thereby limiting recovery. Ultimately, quality of life is notably diminished following critical illness, highlighting the importance of optimal rehabilitation.

\section{PICS-F}

Friends and relatives of critically ill patients experience significant stress, which can lead to profound and enduring psychological disturbance. Over one-quarter will experience clinically significant anxiety, depression and/or PTSD, with one in 10 still exhibiting symptoms at six months. ${ }^{15}$ Sleep deprivation and anticipatory grief, where relatives grapple with feelings of loss before the fact, are both commonly reported. ${ }^{16}$

Following discharge, loved ones often provide informal care for which they have no formal training. The resulting caregiver stress can lead to insufficient self-care,

\section{Box 1. COVID-19 factors increasing risk for PICS and PICS-F}

\section{Cognition ${ }^{18,19}$}

- Potential virus-induced damage to the central nervous system

- Secondary effects of systemic organ damage common in severe illness

- Environmental factors exacerbating delirium

- Depersonalised interactions with staff in frightening personal protective equipment

- Limited human touch

- Isolation from support networks, absence of reorientation and reassurance from loved ones, lack of shared experience

- latrogenic factors

- Prolonged mechanical ventilation, deep sedation, immobilisation

\section{Physical}

- ICU management of severe COVID-19 often involves protracted mechanical ventilation, deep sedation, neuromuscular blockade, proning and prolonged bed rest, increasing risk for: ${ }^{1}$

- ICU-acquired weakness

- diaphragmatic dysfunction

- post-extubation dysphagia

- malnutrition

- Chronic pain is more likely as acute pain is common in COVID-19:9

- painful sequelae of the disease such as myalgia, arthralgia, headache, abdominal and chest pain

- iatrogenic pain from invasive procedures and neuropraxia from proning

- Overstretched inpatient rehabilitation teams potentially delaying crucial early treatment

Psychological

- Patient-caregiver disconnect from infrequent communication or perceived lack of empathy from overburdened staff

- Fear of infecting others, fear of social stigma, uncertainty regarding impact of global pandemic 20

- Survivor's guilt ${ }^{20}$

- Disruptions to hospital religious or pastoral care services

PICS-F'9

- Prolonged separation from patient due to visiting restrictions

- Inability to attend ICU and understand patient's care environment

- Isolation from support systems due to social distancing

- Discussing difficult or distressing subject matter with health professionals via telephone or teleconferencing

- Inability to 'say goodbye' if bereaved, complicating grief

ICU, intensive care unit; PICS, post-intensive care syndrome; PICS-F, post-intensive care syndrome-family 
culminating in physical and psychological morbidity. ${ }^{16}$ This is significant, as effective social support is an important determinant of a patient's post-ICU recovery and whether impairment ultimately results in disability.

\section{COVID-19 pandemic}

The incidences of PICS and PICS-F are anticipated to increase considerably as a result of the COVID-19 pandemic. ${ }^{17}$ Long-term physical and neuropsychological sequelae of COVID-19 are compounded by the prolonged and invasive ICU treatment some survivors endure. Furthermore, infection control measures and restrictions on visitation have changed the ICU environment, with unavoidable negative implications for both patients and their families. Key contributing factors are outlined in Box 1 .

\section{Conclusion}

\section{PICS and PICS-F are debilitating} conditions that carry a heavy personal and health service cost. With the number of ICU survivors rising worldwide, non-ICU clinicians are increasingly participating in the management of these syndromes.

The second article in this series discusses the diagnosis and primary care management of patients with PICS and PICS-F in the COVID-19 era.

\section{Authors}

Pierre Berger MBBS, Intensive Care Registrar, St Vincent's Hospital, Vic

David Braude MBBS, Intensive Care Registrar, Box Hill Hospital, Vic

Competing interests: None.

Funding: None.

Providence and peer review: Not commissioned, externally peer reviewed.

Correspondence to:

pierre.berger1@nhs.net

\section{References}

1. Mikkelsen ME, Netzer G, Iwashyna T. Post-intensive care syndrome (PICS). UpToDate. Available at www.uptodate.com/ contents/post-intensive-care-syndromepics?search $=$ pics \&source=search result\&selectedTitle=1 56\&usage type $=$ default\&display_rank $=1$ [Accessed 18 May 2021].

2. Pandharipande PP, Girard TD, Jackson JC, et al. Long-term cognitive impairment after critical illness. N Engl J Med 2013;369(14):1306-16 doi: 10.1056/NEJMoa1301372.

3. Garcez FB, Apolinario D, Campora F, Curiati JAE, Jacob-Filho W, Avelino-Silva TJ. Delirium and postdischarge dementia: Results from a cohort of older adults without baseline cognitive impairment. Age Ageing 2019;48(6):845-51. doi: 10.1093/ageing/ afz107.

4. Society of Critical Care Medicine. Post-intensive care syndrome. Mount Prospect, IL: Society of Critical Care Medicine, 2013. Available at www. sccm.org/MyICUCare/THRIVE/Post-intensiveCare-Syndrome [Accessed 18 May 2021].

5. Jolley SE, Bunnell AE, Hough CL. ICU-acquired weakness. Chest 2016;150(5):1129-40. doi: 10.1016/j.chest.2016.03.045

6. Modrykamien AM. The ICU follow-up clinic: A new paradigm for intensivists. Respir Care 2012;57(5):764-72. doi: 10.4187/respcare.01461

7. Correia MITD, Waitzberg DL. The impact of malnutrition on morbidity, mortality, length of hospital stay and costs evaluated through a multivariate model analysis. Clin Nutr 2003:22(3):235-39. doi: 10.1016/s02615614(02)00215-7.

8. Orford NR, Lane SE, Bailey M, et al. Changes in bone mineral density in the year after critical illness. Am J Respir Crit Care Med 2016;193(7):736-44. doi: 10.1164/rccm.20150815140C.

9. Kemp HI, Laycock H, Costello A, Brett SJ. Chronic pain in critical care survivors: A narrative review. $\mathrm{Br} J$ Anaesth 2019;123(2):e372-84. doi: 10.1016/j. bja.2019.03.025.

10. Hatch R, Young D, Barber V, Griffiths J, Harrison DA, Watkinson P. Anxiety, depression and post traumatic stress disorder after critical illness: A UK-wide prospective cohort study. Crit Care 2018;22(1):310. doi: 10.1186/s13054-018-2223-6.

11. Kiernan F. Care of ICU survivors in the community: A guide for GPs. Br J Gen Pract 2017;67(663):477-78. doi: 10.3399/ bjgp17X693029.

12. Rovatti KB, Teodoro $M$, de Castro EK. Memories and prevalence of posttraumatic stress disorder in intensive care units. Psicol Reflex Crit 2012;25(3):499-505. Available at www.scielo.br/scielo.php?script=sci arttext\&pid=S0102-79722012000300009 [Accessed 18 May 2021].

13. Harvey MA, Davidson JE. Postintensive care syndrome: Right care, right now ... and later. Crit Care Med 2016;44(2):381-85. doi: 10.1097/ CCM.0000000000001531.

14. Griffiths J, Hatch RA, Bishop J, et al. An exploration of social and economic outcome and associated health-related quality of life after critical illness in general intensive care unit survivors: A 12-month follow-up study. Crit Care 2013;17(3):1-12. doi: 10.1186/cc12745.

15. Davidson JE, Jones $C$, Bienvenu OJ. Family response to critical illness. Crit Care Med 2012;40(2):618-24. doi: 10.1097/CCM.0b013e318236ebf9.

16. Stepanovic K, Van J, Jackson JC. Chapter 5: Family psychological morbidity after the intensive care unit. Netzer G. Families in the intensive care unit. New York City, NY: Springer, 2018.

17. Jackson J, Sevin C. ICU survivorship after COVID19. Nashville, TN: Vanderbilt University Medical Center, 2020. Available at https://discover.vumc. org/2020/05/icu-survivorship-after-covid-19 [Accessed 18 May 2021].

18. Kotfis K, Williams Roberson S, Wilson JE, Dabrowski W, Pun BT, Ely EW. COVID-19: ICU delirium management during SARS-CoV-2 pandemic. Crit Care 2020;24(1):176. doi: 10.1186/ s13054-020-02882-x

19. Waldmann C, Meyer J, Slack A. FICM position statement and provisional guidance: Recovery and rehabilitation for patients following the pandemic. London, UK: Faculty of Intensive Care Medicine, 2020. Available at www.ficm.ac.uk/sites/default/ files/ficm_rehab_provisional_guidance.pdf [Accessed 18 May 2021].

20. Hosey MM, Needham DM. Survivorship after COVID-19 ICU stay. Nat Rev Dis Primers 2020;6(1):60. doi: 10.1038/s41572-020-0201-1. 